\title{
Omega-3 polyunsaturated fatty acids reverse age-related decreases in retinoic acid receptors, retinoid $x$ receptors and peroxisome-proliferator-activated receptors in rat forebrain
}

Simon C. Dyall

Gregory J. Michael

Adina T. Michael-Titus

Neuroscience Centre,

Bart's and the London School of Medicine and Dentistry,

Queen Mary University of London,

Institute of Cell \& Molecular Sciences,

4, Newark Street,

London E1 2AT

Retinoic acid receptors (RAR), retinoid $X$ receptors (RXR) and peroxisome proliferator-activated receptors (PPAR) are transcription factors involved in many cellular processes including learning and memory. These nuclear receptors can be divided into three isotypes: alpha, beta and gamma for RAR and RXR, and alpha, beta/delta and gamma for PPAR. The levels of mRNA for RARs and RXRs decline with ageing, and this decrease can be reversed by treatment with retinoic acid $[1,2]$, which is an agonist at these receptors. Treatment with retinoic acid also alleviates agerelated memory deficits. Omega-3 polyunsaturated fatty acids (PUFA) such as eicosapentaenoic acid (EPA) and docosahexaenoic acid (DHA) also act as endogenous ligands at PPAR and RXR.

We investigated by western blot analysis of rat forebrain if an age-related decline occurred in RAR, RXR and PPAR protein levels, and whether dietary supplementation with EPA and DHA influenced this decline. Twenty-four month-old male Wistar rats were fed a standard diet or a diet supplemented with EPA and DHA for 12 weeks. The average daily intake was $145-160 \mathrm{mg}$ EPA and $95-110 \mathrm{mg} \mathrm{DHA} / \mathrm{kg}$ rat, with an omega-6/omega-3 ratio of 1, provided by the addition of Maxepa ${ }^{\circledR}$ oil to the diet. The control group received the standard diet, with an omega-6/omega-3 ratio of 14 .

With ageing there were decreases in the protein levels of RARalpha (18\%), RARgamma (14\%), RXRalpha (36\%), RXRbeta (47\%) and PPARgamma (40\%). Some of these decreases reached significance $(P<0.05$ vs. adult controls). They were fully reversed by supplementation with omega-3 PUFA. These observations support the neuroprotective potential of omega- 3 fatty acids in ageing and suggest that reversal of age-related changes in retinoid signalling may be involved in their therapeutic effect.

\section{References}

1. Enderlin, et al. Neurosci Lett 1997; 229: 125-9.

2. Etchamendy, et al. J Neurosci 2001; 21: 6423-9.

\section{POSTERS}

